\title{
Linearization of Emden Differential Equation via the Generalized Sundman Transformations
}

\author{
Joel Mvendaga Orverem ${ }^{1,2^{*}}$, Yusuf Haruna², Bala Ma'aji Abdulhamid², Magaji Yunbunga Adamu ${ }^{2}$ \\ ${ }^{1}$ Department of Mathematical Sciences, Federal University Dutsin-Ma, Katsina State, Nigeria \\ ${ }^{2}$ Department of Mathematical Sciences, Abubakar Tafawa Balewa University, Bauchi, Nigeria \\ Email: *orveremjoel@yahoo.com, hayu008@yahoo.com, mbabdulhamid@atbu.edu.ng, magajiadamu78@yahoo.com
}

How to cite this paper: Orverem, J.M., Haruna, Y., Abdulhamid, B.M. and Adamu, M.Y. (2021) Linearization of Emden Differential Equation via the Generalized Sundman Transformations. Advances in Pure Mathematics, 11, 163-168.

https://doi.org/10.4236/apm.2021.113011

Received: January 6, 2021

Accepted: March 9, 2021

Published: March 12, 2021

Copyright $\odot 2021$ by author(s) and Scientific Research Publishing Inc. This work is licensed under the Creative Commons Attribution International License (CC BY 4.0).

http://creativecommons.org/licenses/by/4.0/

\begin{abstract}
The Emden differential equation is one of the most widely studied and challenging nonlinear dynamics equations in literature. It finds applications in various areas of study such as celestial mechanics, fluid mechanics, Steller structure, isothermal gas spheres, thermionic currents and so on. Because of the importance of the equation, the method of generalized Sundman transformation (GST) as proposed by Nakpim and Meleshko is used for linearizing the Emden differential equation. The Emden differential equation considered here is a modification of the equation given by Berkovic. The results obtained in this paper imply that the Emden equation cannot be linearized by a point transformation. The general solution of the modified Emden equation is also obtained.
\end{abstract}

\section{Keywords}

Emden Differential Equation, Second Order, Ordinary Differential Equation, Generalized Sundman Transformation, Linearization

\section{Introduction}

In mathematical physics and astrophysics, Emden differential equations have been used to model many phenomena, such as thermal explosions, Steller structure, spherical gas cloud thermal action, isothermal gas spheres, fluid mechanics and thermionic currents [1].

Mathematically, the Emden equation is an ordinary differential equation of the second order. The Generalized Emden-Fowler equation was considered in [2] where the definition of nonlinear nonautonomous ordinary differential equations of order $n$ with a so-called reducible linear component was considered.

Many people have worked on the Lane-Emden equation. An example is the 
differential Transform System solution of the Lane-Emden equation. See [1] where the authors attempted to extract solutions from one of the commonly studied and challenging nonlinear dynamics equations.

$$
y^{\prime \prime}(x)+\frac{2}{x} y^{\prime}(x)+y^{n}=0
$$

for $n=0,1,2,3,4$ and 5 , by a relatively new exact series solution approach known as the differential transformation method (DTM).

Another approach considered a second-order initial-value problem

$$
y^{\prime \prime}(t)+\frac{2}{t} y^{\prime}(t)+[y(t)]^{n}=0, n \geq 0, y(0)=1, y^{\prime}(0)=0,
$$

which has applications in celestial mechanics and has widely been studied in literature. As can be seen in [3], the known Lane-Emden astrophysics equation is a special case. The authors studied the initial-value problem which is a generalization of Equation (1.2) given as

$$
y^{\prime \prime}(t)+p(t) y^{\prime}(t)+q(t, y(t))=0, t>0, y(0)=a, y^{\prime}(0)=0,
$$

where $a \in \mathbb{R}$ and $p$ may be singular at $t=0$. The local and global existence and the uniqueness of solutions have also been illustrated therein.

For singular initial-value Lane-Emden-type problems, a new analytical method refers to as the residual power series (RPS) is applied, and the effectiveness and performance of the method have been studied in [4]. The Lane-Emden equation is important, a Poisson equation for the gravitational potential of a self-gravitating, symmetrically spherical polytrophic fluid as it is applicable to astrophysics.

Linearization of a class of nonlinear second order Lienard type ordinary differential equations from the point of view of the generalized Sundman transformation (GST) has been studied [5]. In addition, a new characterization of $S$-linearizable equations was studied in terms of ODE coefficients and an auxiliary function, as seen in [6]. The new criteria were used primarily to procure general solutions for the first integral, offering an alternative to constructing the first integrals and transformations of Sundman.

In this paper, we linearize the modified Lane-Emden differential equation using the Generalized Sundman Transformation (GST) given in [7]. To the best of our knowledge, this equation has not been solved via the generalized Sundman transformation in literature before now.

\section{The Generalized Sundman Transformation}

Based on the generalized Sundman transformation taken from [7], we have some key results here. Let us consider the general second order ordinary differential equation of the form

$$
y^{\prime \prime}=f\left(x, y, y^{\prime}\right) .
$$

The formulae defined by 


$$
u(t)=F(x, y), \mathrm{d} t=G(x, y) \mathrm{d} x,\left(G F_{y}\right) \neq 0,
$$

which is a non-point transformation is called a generalized Sundman transformation (GST).

The requisite form of a linearizable ordinary differential Equation (2.1) that can be translated into a linear ordinary differential equation

$$
u^{\prime \prime}+\beta u^{\prime}+\alpha u=\gamma
$$

through the transformation (2.2), is given by

$$
y^{\prime \prime}+f_{2} y^{\prime 2}+f_{1} y^{\prime}+f_{0}=0,
$$

where $\alpha(t), \beta(t)$ and $\gamma(t)$ are some functions.

Consider the case $f_{3} \neq 0$ and $f_{5} \neq 0$, where $f_{3}=f_{1 y}-2 f_{2 x}$, $f_{4}=2 f_{0 y y}-2 f_{1 x y}+2 f_{0} f_{2 y}-f_{1 y} f_{1}+2 f_{0 y} f_{2}+2 f_{2 x x}$ and $f_{5}=f_{2 x x}+f_{2 x} f_{1}+f_{3 x}+f_{1} f_{3}$. For the Equation (2.4) to be linearizable by (2.2), the sufficient conditions are:

$$
\begin{gathered}
f_{0 x}=2 f_{0} \frac{\left(-f_{1} f_{3}+f_{5}\right)}{f_{3}} \\
f_{2 x x y}=-f_{2 x y} f_{1}-f_{3 x y}-2 f_{2 x}^{2}-2 f_{2 x} f_{3}-f_{3 y} f_{1}+\left(f_{3 y} f_{5}\right) f_{3}^{-1}, \\
f_{2 x x x}=-f_{3 x x}-f_{1 x} f_{2 x}-f_{1 x} f_{3}+f_{2 x} f_{1}^{2}+f_{1}^{2} f_{3}-2 f_{1} f_{5}+f_{3}^{-1} f_{5}\left(f_{3 x}+f_{5}\right),
\end{gathered}
$$

and

$$
\begin{aligned}
& f_{3} f_{5}\left(6 f_{0 y} f_{2 x}+2 f_{2 x y} f_{0}+4 f_{2 x} f_{0} f_{2}+2 f_{3 y} f_{0}+4 f_{0} f_{2} f_{3}+f_{1} f_{5}\right) \\
& -f_{3}^{2}\left(6 f_{2 x}^{2} f_{0}+12 f_{2 x} f_{1} f_{3}-6 f_{0 y} f_{5}+6 f_{0} f_{3}^{2}\right)-f_{4} f_{5}^{2}-2 f_{5}^{3}=0 .
\end{aligned}
$$

One obtains the functions $F$ and $G$ by solving the following equations:

$$
\begin{gathered}
F_{x}=0, \\
F_{y y}=\frac{F_{y} G_{y}+f_{2} F_{y} G}{G}, \\
G_{x}=\frac{G\left(f_{2 x x}+f_{2 x} f_{1}+f_{3 x}\right)}{f_{3}}, \\
G_{y}=\frac{G f_{3}\left(f_{2 x}+f_{3}\right)}{f_{5}} .
\end{gathered}
$$

The constants from (2.3), $\alpha, \beta$ and $\gamma$ are determined from the equations

$$
\begin{gathered}
\alpha=\frac{G\left(f_{0 y}+f_{0} f_{2}\right)-G_{y} f_{0}}{G^{3}}, \\
\beta=\frac{G_{x}+G f_{1}}{G^{2}}, \\
\gamma=\frac{\alpha F G^{2}-F_{y} f_{0}}{G^{2}} .
\end{gathered}
$$

\section{Linearization of Emden Differential Equation via GST}

The general Lane-Emden equation of second order as given in [2] is 


$$
y^{\prime \prime}+a_{1}(x) y^{\prime}+a_{0}(x) y+f(x) y^{n}=0, n \neq 0, n \neq 1 .
$$

Taking the coefficients from (3.1) to be

$$
a_{1}(x)=a y, a_{0}(x)=0, f(x)=\frac{a^{2}}{9}, n=3,
$$

we have that

$$
y^{\prime \prime}+a y y^{\prime}+\frac{a^{2}}{9} y^{3}=0 .
$$

Equation (3.2) is the modified Lane-Emdem differential equation that we want to linearize here.

From (3.2), we see that $f_{2}=0, f_{1}=a y, f_{0}=\frac{a^{2}}{9} y^{3}$. Again $f_{3}=a, f_{4}=\frac{a^{2} y}{3}$ and $f_{5}=a^{2} y$.

By a survey, we see that Equations (2.5), (2.6), (2.7) and (2.8) are satisfied. Thus, through the generalized Sundman transformation, the Equation (3.2) is linearizable.

From the Equations (2.9), (2.10), (2.11) and (2.12), the functions $F$ and $G$ are found to be

$$
\begin{gathered}
F_{x}=0, G_{x}=0, \\
G_{y}=\frac{G}{y}, \\
F_{y y}=\frac{F_{y}}{y} .
\end{gathered}
$$

The solution $F=y^{2}$ and $G=y$ satisfies Equations (3.3), (3.4) and (3.5). We can now obtain the transformation from (2.2) as

$$
u=y^{2}, \mathrm{~d} t=y \mathrm{~d} x .
$$

Equations (2.13), (2.14) and (2.15) give

$$
\begin{gathered}
\alpha=\frac{2 a^{2}}{9}, \\
\beta=a, \\
\gamma=0 .
\end{gathered}
$$

Hence, Equation (2.3) becomes

$$
u^{\prime \prime}+a u^{\prime}+\frac{2 a^{2}}{9} u=0 .
$$

It is necessary to obtain the general solution of (3.10) to be

$$
u=c_{1} \mathrm{e}^{-\frac{a t}{3}}+c_{2} \mathrm{e}^{-\frac{2 a t}{3}},
$$

where $c_{1}, c_{2}$ are arbitrary constants. We apply the GST (3.6) to the Emden Equation (3.2) to obtain the general solution of the Emden Equation (3.2) as

$$
y(x)=\sqrt{c_{1} \mathrm{e}^{-\frac{a}{3} \phi(x)}+c_{2} \mathrm{e}^{-\frac{2 a}{3} \phi(x)}} .
$$


The function $t=\phi(x)$ is a solution of the equation

$$
\frac{\mathrm{d} t}{\mathrm{~d} x}=\sqrt{c_{1} \mathrm{e}^{-\frac{a}{3} t}+c_{2} \mathrm{e}^{-\frac{2 a}{3} t}} .
$$

\section{Remark}

Recall the results on linearization via the differential forms as in [8] and [9] where a point transformation was considered. To be linearizable, the required equation form of (2.1) is

$$
y^{\prime \prime}+f_{0}+f_{1} y^{\prime}+f_{2} y^{\prime 2}+f_{3} y^{\prime 3}=0 .
$$

Equation (4.1) is linearizable if its coefficients satisfy the sufficient linearizability conditions

$$
f_{0 y y}+f_{0}\left(f_{2 y}-2 f_{3 x}\right)+f_{2} f_{0 y}-f_{3} f_{0 x}+\left(\frac{1}{3}\right)\left(f_{2 x x}-2 f_{1 x y}+f_{1} f_{2 x}-2 f_{1} f_{1 y}\right)=0
$$

and

$$
f_{3 x x}+f_{3}\left(2 f_{0 y}-f_{1 x}\right)+f_{0} f_{3 y}-f_{1} f_{3 x}+\left(\frac{1}{3}\right)\left(f_{1 y y}-2 f_{2 x y}+2 f_{2} f_{2 x}-f_{2} f_{1 y}\right)=0 .
$$

Though the form (2.4) is a special case of (4.1), appropriate linearization conditions through the point transformations (4.2) and (4.3), and the generalized transformation of Sundman are different. On a check, one sees that the coefficients of Equation (3.2) do not satisfy equations and (4.3) and hence, not linearizable via differential forms (point transformation).

\section{Conclusion}

In this research, the modified Emden differential equation is linearized using the generalized Sundman transformation method. The authors went ahead to obtain the general solution of the modified Emden differential equation. We also demonstrate that, though the modified equation $y^{\prime \prime}+a y y^{\prime}+\frac{a^{2}}{9} y^{3}=0$ is a special form of equation $y^{\prime \prime}+f_{0}+f_{1} y^{\prime}+f_{2} y^{\prime 2}+f_{3} y^{\prime 3}=0$, where $f_{3}=0$, but the coefficients of $y^{\prime \prime}+a y y^{\prime}+\frac{a^{2}}{9} y^{3}=0$ does not satisfy the linearizability conditions

$$
f_{0 y y}+f_{0}\left(f_{2 y}-2 f_{3 x}\right)+f_{2} f_{0 y}-f_{3} f_{0 x}+\left(\frac{1}{3}\right)\left(f_{2 x x}-2 f_{1 x y}+f_{1} f_{2 x}-2 f_{1} f_{1 y}\right)=0
$$

and

$$
f_{3 x x}+f_{3}\left(2 f_{0 y}-f_{1 x}\right)+f_{0} f_{3 y}-f_{1} f_{3 x}+\left(\frac{1}{3}\right)\left(f_{1 y y}-2 f_{2 x y}+2 f_{2} f_{2 x}-f_{2} f_{1 y}\right)=0,
$$

and hence not linearizable via point transformation (differential forms).

\section{Conflicts of Interest}

The authors declare no conflicts of interest regarding the publication of this paper. 


\section{References}

[1] Mukherjee, S., Roy, B. and Chaterjee, P.K. (2011) Solution of Lane-Emden Equation by Differential Transform Method. International Journal of Nonlinear Science, 12, 478-484.

[2] Berkovic, L.M. (1997) Generalized Emden-Fowler Equation. Symmetry in Nonlinear Mathematical Physics, 1, 155-163.

[3] Aslanov, A. (2008) A Generalization of the Lane-Emden Equation. International Journal of Computer Mathematics, 85, 1709-1725. https://doi.org/10.1080/00207160701558457

[4] Abu Arqub, O., El-Ajou, A., Bataineh, A.S. and Hashim, I. (2013) A Representation of the Exact Solution of Generalized Lane-Emden Equations Using a New Analytical Method. Abstract and Applied Analysis, 2013, Article ID: 378593. https://doi.org/10.1155/2013/378593

[5] Johnpillai, A.G. and Mahomed, F.M. (2013) On Linearization by Generalized Sundman Transformations of a Class of Liénard Type Equations and Its Generalization. Applied Mathematics \& Information Sciences, 7, 2355-2359. https://doi.org/10.12785/amis/070627

[6] Mustafa, M.T., Al-Dweik, A.Y. and Mara'beh, R.A. (2013) On the Linearization of Second-Order Ordinary Differential Equations to the Laguerre Form via Generalized Sundman Transformations. Symmetry, Integrability and Geometry. Methods and Applications (SIGMA), 9, 1-10. https://doi.org/10.3842/SIGMA.2013.041

[7] Nakpim, W. and Meleshko, S.V. (2010) Linearization of Second-Order Ordinary Differential Equations by Generalized Sundman Transformations. Symmetry, Integrability and Geometry: Methods and Applications (SIGMA), 6, 1-11. https://doi.org/10.3842/SIGMA.2010.051

[8] Harrison, B.K. (2002) An Old Problem Newly Treated with Differential Forms: When and How Can the Equation $\mathrm{y}=\mathrm{f}(\mathrm{x}, \mathrm{y}, \mathrm{y})$ Be Linearized ? Proceedings of Institute of Mathematics of NAS of Ukraine, 43, 27-35.

[9] Orverem, J.M., Tyokyaa, R.K. and Balami, H.M. (2017) Differential Forms : A Tool for Linearizing Second Order Ordinary Differential Equations. Journal of Scientific and Engineering Research, 4, 213-219. 